\title{
O oscilador harmónico
}

\section{CITAÇÃO}

Lage, E. (2019)

O oscilador harmónico,

Rev. Ciência Elem., V7 (02):033

doi.org/10.24927/rce2019.033

\section{EDITOR}

José Ferreira Gomes,

Universidade do Porto

\section{EDITOR CONVIDADO}

Paulo Fonseca,

Universidade de Lisboa

\section{RECEBIDO EM}

05 de junho de 2019

\section{ACEITE EM}

05 de junho de 2019

\section{PUBLICADO EM}

21 de junho de 2019

\section{COPYRIGHT}

(C) Casa das Ciências 2019.

Este artigo é de acesso livre, distribuído sob licença Creative Commons com a designação CC-BY-NC-SA 4.0, que permite a utilização e a partilha para fins não comerciais, desde que citado o autor e a fonte original do artigo.

rce.casadasciencias.org

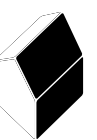

\author{
Eduardo Lage \\ Universidade do Porto \\ ejslage@gmail.com
}

O oscilador harmónico está omnipresente em Física porque é um modelo simples para fenómenos simplesmente periódicos. Aqui é estudado como objeto mecânico, o que permitirá estender muitas dos resultados ao eletromagnetismo, teoria da elasticidade, dinâmica de fluidos e, mais genericamente, ao estudo de ondas.

O oscilador harmónico é o dispositivo mais simples capaz de exibir um fenómeno periódico, ainda mais simples que um pêndulo, pois que este àquele se reduz para pequenas amplitudes de oscilação. Experimentalmente, é fácil construir um oscilador: uma mola suspensa verticalmente, presa no extremo superior e tendo uma pequena massa agarrada à extremidade inferior, como se exibe na FIGURA 1. Esticando a mola, aquela massa entra em oscilações periódicas tornando possível estudar e medir muitas das propriedades que, aqui, serão apresentadas.

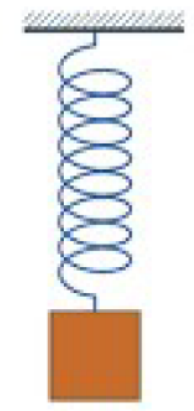

FIGURA 1. Oscilador harmónico: uma massa suspensa por uma mola.

Mas o oscilador harmónico é, também, um modelo simples para a descrição de muitos fenómenos em Física. Numa molécula diatómica, por exemplo, os átomos interagem através de um potencial que apresenta um mínimo (ver FIGURA 1 em "Equação de estado de Van der Waals"), pelo que, para energias ligeiramente superiores ao mínimo, a molécula comporta-se como um oscilador harmónico. E mesmo uma molécula poliatómica pode ser modelizada como uma coleção de osciladores harmónicos, designados por modos normais de vibração, quando a energia mecânica da molécula, soma das 
energias cinéticas dos seus átomos com as energias de interação entre os átomos, se situa próxima do seu mínimo. 0 mesmo se passa num sólido que, para os efeitos aqui descritos, pode ser visualizado como uma molécula gigante. Um outro exemplo é um circuito LC, uma bobina e um condensador eletricamente conectados: o condensador descarrega carga e a consequente corrente elétrica induz uma f.e.m. na bobina, originando, assim, uma oscilação harmónica da carga e da corrente, facilmente mensuráveis por um voltímetro e um amperímetro, respetivamente. Outros exemplos surgem do comportamento elástico de materiais: uma barra horizontal, encastrada numa extremidade, apresentará oscilações harmónicas quando posta a vibrar verticalmente; um fio metálico, suspenso na vertical, também oscilará harmonicamente quando é torcido por aplicação de um momento na sua base inferior; e o mesmo fio, preso nas suas extremidades e mantido tenso, é sede de oscilações que se propagam (ver "Campos").

Estudemos, agora, o oscilador harmónico, considerando, apenas, o caso unidimensional que servirá para apresentar os conceitos e resultados mais fundamentais. Designemos por $x=0$ a posição de equilíbrio do oscilador; afastada desta origem, a massa $m$ é atuada pela força $-k x$, onde o sinal exibe claramente que a massa é sempre puxada para a origem, quer quando a mola é esticada $(x>0)$, quer quando é comprimida $(x<0)$. A constante $k$ é conhecida por constante da mola ou, mais geralmente, do oscilador. Se além desta força, designada por harmónica, existir uma força $f(t)$ externamente aplicada ao oscilador, a equação de movimento fica:

$$
m \frac{d^{2} x}{d t^{2}}=-k x+f(t)
$$

Trata-se de uma equação linear pelo que é válido o princípio da sobreposição: a soma de soluções é, ainda, uma solução. Assim, podemos estudar separadamente vários casos de interesse. Mais adiante, acrescentaremos o efeito do atrito (de Stokes), originando amortecimento, mas mantendo a linearidade da equação.

$\mathrm{Na}$ ausência de forças externas $(f=0)$, a solução da eq. (1) exprime-se, simplesmente, à custa de funções trigonométricas:

$$
m \frac{d^{2} x}{d t^{2}}+k x=0 \quad \rightarrow \quad x(t)=A \cos \left(\omega_{0} t\right)+B \operatorname{sen}\left(\omega_{0} t\right)
$$

onde $\omega_{0}=\sqrt{\frac{k}{m}}$ é a frequência (angular) própria do oscilador. As constantes A e B são determinadas pelas condições iniciais: designando por $x_{0}$ e $v_{0}$, respetivamente, a posição e velocidade iniciais, obtem-se:

$$
\begin{gathered}
x_{0} \equiv x(0)=A \\
v_{0} \equiv \dot{x}(0)=\omega_{0} B
\end{gathered}
$$

Este é o movimento natural do oscilador, i.e., não sujeito a ações externas. Consideremos, agora, o efeito de forças externas. Para isso, torna-se muito mais simples e útil estudarmos o complexo $z(t) \equiv x(t)+i \frac{v(t)}{\omega_{0}}$. Se conhecermos $z(t)$, então a sua parte real é a posição atual $x(t)$ e da sua parte imaginária deduzimos a velocidade $v(t)$ do oscilador. Ora:

$$
\frac{d z}{d t}=v(t)+\frac{i}{\omega_{0}} \frac{d v}{d t}=v(t)+\frac{i}{m \omega_{0}}(-k x+f(t))
$$


onde empregamos a eq. (1). Substituindo $k=m \omega_{0}^{2}$, encontramos:

$$
\frac{d z}{d t}=v(t)-i \omega_{0} x(t)+\frac{i}{m \omega_{0}} f(t)=-i \omega_{0} z(t)+\frac{i}{m \omega_{0}} f(t)
$$

Isto é:

$$
\frac{d z}{d t}+i \omega_{0} z(t)=\frac{i}{m \omega_{0}} f(t)
$$

Esta equação é linear, pelo que se aplica o princípio da sobreposição. A solução da equação homogénea é imediata:

$$
\frac{d z}{d t}+i \omega_{0} z(t)=0 \quad \rightarrow \quad z(t)=z_{0} e^{-i \omega_{0} t}
$$

onde $z_{0}$ é uma constante (complexa). Esta solução é o movimento natural, atrás analisado. Para, agora, encontrarmos uma solução particular da eq. (3), basta considerar $z(t)=w(t) e^{-i \omega_{0} t}$. Substituindo na eq. (3), obtemos:

$$
\frac{d w}{d t}=\frac{i}{m \omega_{0}} e^{i \omega 0 t} f(t) \quad \rightarrow \quad w(t)=z_{0}+\frac{i}{m \omega_{0}} \int_{0}^{t} d t^{\prime} e^{i \omega t^{\prime}} f\left(t^{\prime}\right)
$$

Assim, a solução geral da eq. (3) escreve-se:

$$
z(t)=z_{0} e^{-i \omega_{0} t}+\frac{i}{m \omega_{0}} \int_{0}^{t} d t^{\prime} e^{i \omega_{0}\left(t^{\prime}-t\right)} f\left(t^{\prime}\right)
$$

0 complexo $z_{0}$ é inteiramente determinado pelas condições iniciais:

$$
z_{0}=z(0)=x(0)+\frac{i}{\omega_{0}} v(0)
$$

No que se segue, suporemos $z_{0}=0$, concentrando-nos, pois, apenas no efeito da força aplicada.

a. Comecemos por considerar o caso da força exterior constante $\left(f_{0}\right)$, aplicada no instante inicial - é designada por força súbita. A solução da eq. (4) é:

$$
z(t)=\frac{i f_{0}}{n \omega_{0}} \int_{0}^{t} d t^{\prime} e^{i \omega_{0}\left(t^{-}-t\right)}=\frac{f_{0}}{m \omega_{0}^{2}}\left(1-e^{-i \omega_{0} t}\right)
$$

Assim:

$$
\begin{gathered}
x(t)=\operatorname{Re}[z(t)]=\frac{f_{0}}{m \omega_{0}^{2}}\left(1-\cos \left(\omega_{0} t\right)\right) \\
v(t)=\operatorname{Im}[z(t)]=\frac{f_{0}}{m \omega_{0}} \operatorname{sen}\left(\omega_{0} t\right)
\end{gathered}
$$

Ooscilador desloca-se harmonicamente entrea origemea máxima amplitude $x=\frac{2 f_{0}}{m \omega_{0}^{2}}=\frac{2 f}{k}$

b. Um segundo caso de interesse consiste em admitir que a força externa cresce muito lentamente até atingir o valor limite $f_{0}$ - é designada por força adiabática. Uma expressão analítica para tal força é $f(t)=f_{0}\left(1-e^{-\varepsilon t}\right)$, onde se considera $\varepsilon \rightarrow 0$ no fim dos cálculos, pelo que o tempo para a força atingir o seu valor limite é da ordem de $\frac{1}{\varepsilon} \rightarrow \infty$. Inserindo na eq. (4), obtemos: 
$z(t)=\frac{i f_{0}}{m \omega_{0}} \int_{0}^{t} d t^{\prime} e^{i \omega_{0}\left(t^{\prime}-t\right)}\left(1-e^{-\varepsilon t^{\prime}}\right)=\frac{i f_{0}}{m \omega_{0}}\left\{\left[\frac{1}{i \omega_{0}}-\frac{1}{i \omega_{0}-\varepsilon}\right]\left[1-e^{i \omega_{0} t}\right]+\frac{1-e^{-\varepsilon t}}{i \omega_{0}-\varepsilon}\right\}$

Tomando o limite $\varepsilon \rightarrow 0$, vem:

$$
z(t)=\frac{f_{0}}{m \omega_{0}^{2}}=\frac{f_{0}}{k}
$$

Vemos, assim, que a força adiabática reproduz a ideia de se manter, praticamente, o oscilador sempre em equilíbrio, levando-o até à posição final desejada, $x=\operatorname{Re}[z(t)]=\frac{f_{0}}{k}$, com velocidade nula, $v(t)=\operatorname{Im}[z(t)]=0$. Deste modo, o trabalho realizado pela força adiabática aparece como energia potencial elástica, $V=\frac{1}{2} k x^{2}$, sendo imediato verificar que a energia mecânica $E=\frac{1}{2} m v^{2}+V(x)$ se conserva na ausência de forças exteriores.

c. Um terceiro exemplo considera a resposta do oscilador a uma força exteriorperiódica, i.e., $f(t)=f_{0} \cos (\omega t)$. Por resposta, entende-se a solução particular da eq. (3), sendo fácil verificar que tal solução é:

$$
x(t)=\frac{f_{0}}{m\left(\omega_{0}^{2}-\omega^{2}\right)} \cos (\omega t)
$$

Este importante resultado aparece em muitos domínios da Física, por exemplo na resposta de um dipólo elétrico a um campo elétrico aplicado; neste contexto, a eq. (5) fornece, diretamente, a suscetibilidade elétrica. Não escapará ao leitor que esta solução apresenta uma divergência quando a frequência da força aplicada $(\omega)$ iguala a frequência própria $\left(\omega_{0}\right)$ do oscilador, situação conhecida por ressonância. Podemos ver o que se passa regressando à eq. (4) para obter a resposta à força $f(t)=f_{0} \cos \left(\omega_{0} t\right)$. Assim:

$$
z(t)=\frac{i f_{0}}{2 m \omega_{0}} \int_{0}^{t} d t^{\prime} e^{i \omega_{0}\left(t^{\prime}-t\right)}\left(e^{i \omega_{0} t^{\prime}}+e^{-i \omega_{0} t^{\prime}}\right)=\frac{f_{0}}{2 m \omega_{0}}\left(\mathrm{i} \frac{\operatorname{sen}\left(\omega_{0} t\right)}{\omega_{0}}+t \cos \left(\omega_{0} t\right)\right)
$$

Assim:

$$
\begin{gathered}
x(t)=\frac{f_{0}}{2 m \omega_{0}} t \operatorname{sen}\left(\omega_{0} t\right) \\
v(t) \frac{f_{0}}{2 m \omega_{0}}\left(\operatorname{sen}\left(\omega_{0} t\right)+t \cos \left(\omega_{0} t\right)\right)
\end{gathered}
$$

Na ressonância, posição e velocidade cresceriam sem limitações.

Na prática, esta divergência não ocorre porque não podemos ignorar efeitos dissipativos tais como uma força de Stokes no meio viscoso onde o oscilador se move. Tal obriga-nos a acrescentar esta força ao $2^{\circ}$ membro da eq. (1), que escreveremos sob a forma $-m \gamma v$, obtendo-se ${ }^{1}$ :

$$
\frac{d^{2} x}{d t^{2}}+\gamma \frac{d x}{d t}+\omega_{0}^{2} x=\frac{f(t)}{m}
$$

\footnotetext{
${ }^{1}$ Esta equação é formalmente idêntica à de um circuito RLC, com a carga do condensador a desempenhar o papel da posição x. 0 circuito pode, então, considerar-se um computador analógico para o oscilador amortecido.
} 
Procuremos a solução particular para $f(t)=f_{0} \cos (\omega t)=R e\left[f_{0} e^{i \omega t}\right]$, a qual terá a forma $x(t)=\operatorname{Re}\left[a(\omega) e^{i \omega t}\right]$. Obtemos:

$$
a(\omega)=\frac{f_{0}}{m} \frac{1}{\omega_{0}^{2}-\omega^{2}+i \gamma \omega}
$$

Esta resposta não é mais divergente na ressonância; ela origina uma suscetibilidade complexa nos materiais dielétricos, por exemplo. A FIGURA 2 mostra as partes real e imaginária de $\alpha(y) \equiv \frac{m \omega_{0}^{2}}{f_{0}} a(\omega)$, com $y \equiv \frac{\omega}{\omega_{0}}$, para fraco amortecimento, $\frac{\gamma}{\omega_{0}}=0.1$. São evidentes os picos acentuados da parte imaginária na ressonância. A figura seguinte exibe as mesmas grandezas na situação de forte amortecimento, $\frac{\gamma}{\omega_{0}}=10$.

A)

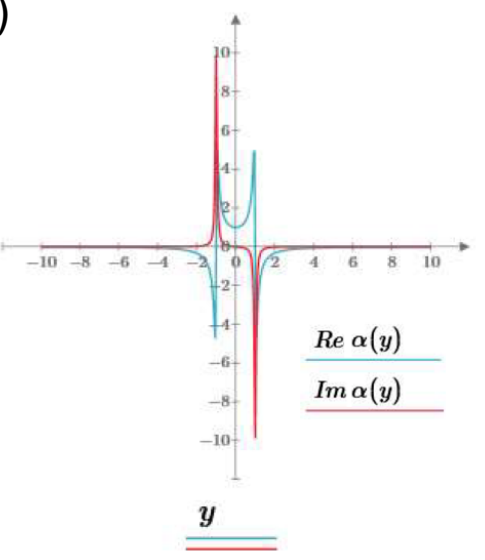

B)

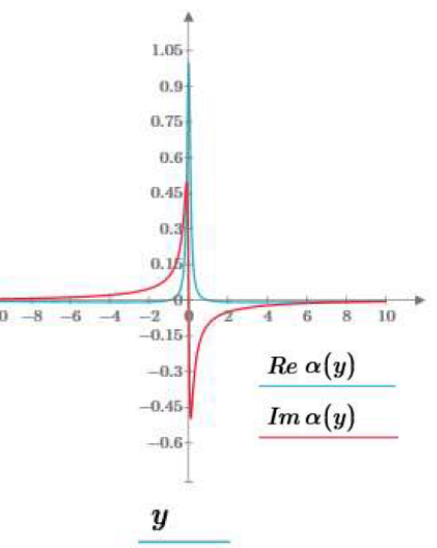

FIGURA 2. A) Partes real e imaginária de $\alpha(y)$ para fraco amortecimento. B) para forte amortecimento.

Podemos ver mais claramente a relação entre amortecimento e dissipação da energia fornecida pela força aplicada, analisando como varia a energia mecânica, atrás escrita, ao longo do tempo. Tem-se:

$$
\frac{d E}{d t}=m v\left(\frac{d^{2} x}{d t^{2}}+\omega_{0}^{2} x\right)
$$

Usando, agora, a eq. (6), obtemos:

$$
\frac{d E}{d t}=f(t) v(t)-\gamma m v(t)^{2}
$$

Efetuemos a média desta equação sobre um período da força aplicada. A média do 1o membro é nula, porque posição e velocidade do oscilador regressam aos mesmos respetivos valores ao fim de um ciclo. Deste modo, a média da potência da força é igual à energia dissipada, i.e., $\langle f(t) v(t)\rangle=\gamma m\left\langle(v(t))^{2}\right\rangle$.

Ora,

$$
v(t)=\frac{d x}{d t}=\operatorname{Re}\left[i \omega a(\omega) e^{i \omega t}\right]=\omega[\operatorname{Re}[a(\omega)] \operatorname{sen}(\omega t)+\operatorname{Im}[a(\omega)] \cos (\omega t)]
$$

pelo que $\langle f(t) v(t)\rangle=-\frac{f_{0} \omega}{2} \operatorname{Im}[a(\omega)]=\frac{f_{0}^{2}}{2 m} \frac{\gamma \omega^{2}}{\left(\omega_{0}^{2}-\omega^{2}\right)^{2}+\gamma^{2} \omega^{2}} \geq 0$.

Dissipação e parte imaginária da resposta (suscetibilidade) estão, pois, intimamente re- 
lacionadas.

Por último, podemos analisar o comportamento do oscilador, livre de forças externas, mas sujeito a dissipação, i.e., procurar as soluções da eq. (6) com $2^{\circ}$ membro nulo.

Não são necessários cálculos adicionais: se existem soluções com $f_{0}=0$, então, observando a eq. (7), também deverá ser nulo o denominador no $2^{0}$ membro desta equação, obtendo-se, pois, as frequências naturais do oscilador:

$$
\omega_{0}^{2}-\omega^{2}+i \gamma \omega=0 \rightarrow \frac{\omega_{ \pm}}{\omega_{0}}=\frac{i \gamma}{2 \omega_{0}} \pm \sqrt{1-\left(\frac{\gamma}{2 \omega_{0}}\right)^{2}}
$$

Assim, $x(t)=\operatorname{Re}\left[A_{+} e^{i \omega+t}+A_{-} e^{i \omega-t}\right]$ com as constantes $A_{ \pm}$determinadas pelas condições iniciais. Para fraco amortecimento, $\frac{\gamma}{\omega_{0}} \ll 1$, obtém-se $\frac{\omega_{ \pm}}{\omega_{0}}=\frac{i \gamma}{2 \omega_{0}} \pm 1$ que, substituídos em $x(t)$, originam $x(t)=e^{-\frac{\gamma t}{2}} \operatorname{Re}\left[A_{+} e^{i \omega_{0} t}+A_{-} e^{i \omega_{0} t}\right]$ : as oscilações, têm a frequência própria, mas amortecem para 0 , como se mostra na FIGURA 3.

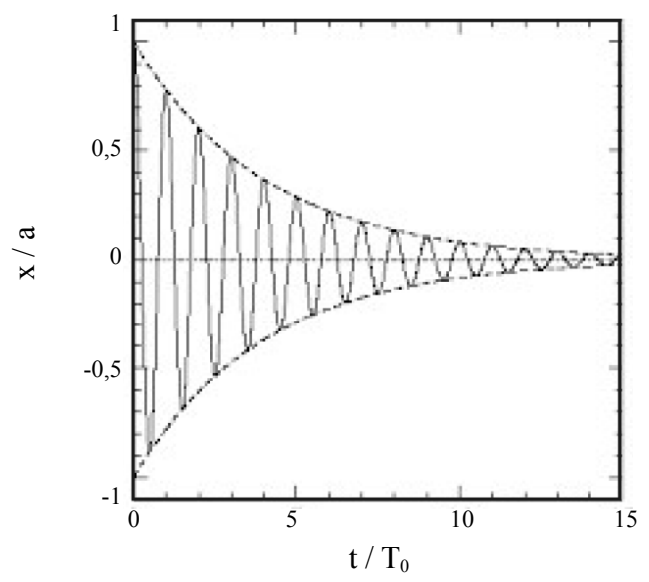

FIGURA 3. Oscilação amortecida

Para forte amortecimento, $\frac{\gamma}{\omega_{0}} \gg 1$, não há qualquer oscilação: $\omega_{+} \simeq i \gamma$ e $\omega_{-} \simeq \frac{i \omega_{0}^{2}}{\gamma}$, $x(t)=\operatorname{Re}\left[A_{+} e^{-\gamma t}+A_{-} e^{\frac{\omega_{0}^{2} t}{\gamma}} \underset{t \gg \frac{1}{\gamma}}{T} R e\left[A_{-} e^{-\frac{\omega_{0}^{2} t}{\gamma}}\right]\right.$. A transição de amortecimento fraco para amortecimento forte dá-se para $\gamma=2 \omega_{0}-$ é o amortecimento crítico. Neste $x(t)=e^{-\frac{\gamma t}{2}}\left[x_{0}+\left(v_{0}+\frac{\gamma x_{0}}{2}\right) t\right]$, sendo este o mais rápido decaímento, para a origem, do oscilador, nas mesmas condições iniciais.

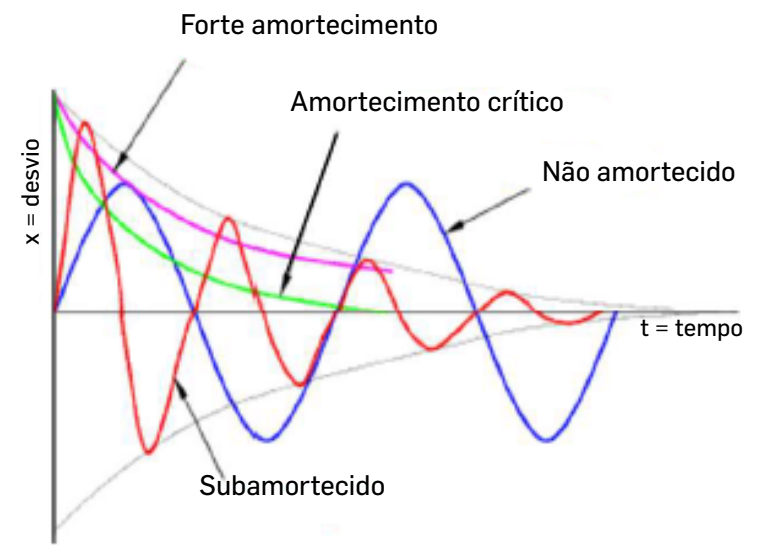




\section{REVISTA DE CIÊNCIA ELEMENTAR}

A FIGURA 4 resume todos os comportamentos do oscilador livre.

\section{REFERÊNCIAS}

${ }^{1}$ FEYNMAN, R.P. et al. The Feynman Lectures on Physics. San Francisco: Pearson/Addison-Wesley ,Vol. 1, section 12-5, 2006. ISBN 0-8053-9049-9

${ }^{2}$ GOLDSTEIN, H. et al. Classical Mechanics, Addison Wesley 3ª edição, 2001.

${ }^{3}$ KIBBLE, T. W. B. \& BERKSHIRE, F. H. , Classical Mechanics, 1966. 